\title{
Quantum and semiclassical oscillations in the organic metal (BEDO-TTF) ${ }_{2} \mathrm{Cl}_{x}\left(\mathrm{H}_{2} \mathrm{O}\right)_{y}$
}

\author{
S. I. Pesotskiï*)
}

Institute of Chemical Physics, Russian Academy of Sciences, 142432 Chernogolovka, Moscow Region, Russia;

International High Magnetic Field and Low temperature Laboratory, 53-529 Wroclaw, Poland; Walther-Meissner Institute, D-85748 Garching, Germany

R. B. Lyubovskiı̌

Institute of Chemical Physics, Russian Academy of Sciences, 142432 Chernogolovka, Moscow Region, Russia;

International High Magnetic Field and Low Temperature Laboratory, 53-529 Wroclaw, Poland

P. Behm, W. Biberacher, and K. Andres

Walther-Meissner Institute, D-85748 Garching, Germany

M. V. Kartsovnik

Walther-Meissner Institute, D-85748 Garching, Germany;

Institute of Solid State Physics, Russian Academy of Sciences, 142432 Chernogolovka, Moscow Region, Russia

\section{A. E. Kovalev and T. G. Togonidze}

Institute of Solid State Physics, Russian Academy of Sciences, 142432 Chernogolovka, Moscow Region, Russian

J. A. A. J. Perenboom

High Field Magnetic Laboratory, Catholic University, NL 6525, ED Nijmegen, Netherlands

N. D. Kushch, R. N. Lyubovskaya, and E. I. Zhilyaeva

Institute of Chemical Physics, Russian Academy of Sciences, 142432 Chernogolovka, Moscow Region, Russia

(Submitted 3 March 1998)

Zh. Éksp. Teor. Fiz. 114, 1137-1146 (September 1998)

We study quantum (Shubnikov-de Haas and de Haas-van Alphen) oscillations and angular oscillations of the reluctance in the organic quasi-two-dimensional metal $(\mathrm{BO})_{2} \mathrm{Cl}_{x}\left(\mathrm{H}_{2} \mathrm{O}\right)_{y}$. We show that the Fermi surface in this compound consists of a slightly corrugated cylinder with its axis perpendicular to the conducting plane. The cross section of the cylinder in this plane is a perfect circle of radius $k_{F} \simeq 3 \times 10^{7} \mathrm{~cm}^{-1}$. The effective carrier mass associated with this cylinder is $m^{*}=(1.65-2.0) m_{0}$ in the conducting plane, while the Dingle temperature is $T_{D}=3-4 \mathrm{~K}$. (C) 1998 American Institute of Physics. [S1063-7761(98)02709-7]

\section{INTRODUCTION}

The compound $(\mathrm{BO})_{2} \mathrm{Cl}_{x}\left(\mathrm{H}_{2} \mathrm{O}\right)_{y}$ belongs to the class of quasi-two-dimensional organic metals. The major structural material in this compound, which forms the conducting layers, is the organic molecule $\mathrm{BO}=(\mathrm{BEDO}-\mathrm{TTF})$ (bis(ethylenedioxy)-tetrathiofulvalene), an analog of the well known ET molecule used as a basis for synthesizing the overwhelming majority of quasi-two-dimensional organic metals and superconductors. ${ }^{1}$ Unlike the ET molecule, in the BO molecule the peripheral atoms of sulfur are replaced by oxygen atoms. Since overlap of the orbitals of these sulfur atoms is responsible for the high conductivity in a layer made up of ET molecules, one might expect substantial changes in the conducting properties on going to metals based on $\mathrm{BO}$.
The first report on the synthesis, structure, and properties of the metallic chloride of $\mathrm{BO}$ contained the chemical formula of this compound, $(\mathrm{BO}) \mathrm{Cl}\left(\mathrm{H}_{2} \mathrm{O}\right)$, a simple salt. ${ }^{2}$ The simple salt is characterized by a $1: 1$ ratio of the $\mathrm{BO}$ cation to the $\mathrm{Cl}\left(\mathrm{H}_{2} \mathrm{O}\right)$ anion, which corresponds to the transfer of an electron from each $\mathrm{BO}$ molecule to an anion and implies a half-filled metal band. This report was of great interest, since before then no organic metals with a half-filled conduction band were known.

The next paper $^{3}$ showed that the chemical formula of the BO chloride was more properly written in the form of the complex salt $(\mathrm{BO})_{2} \mathrm{Cl}\left(\mathrm{H}_{2} \mathrm{O}\right)_{3}$. In this case, one electron arrives at an ion from every two $\mathrm{BO}$ molecules, and the conduction band is then one-quarter full. A comparison of theoretical calculations of the structure of $\mathrm{BO}$ chloride with the 


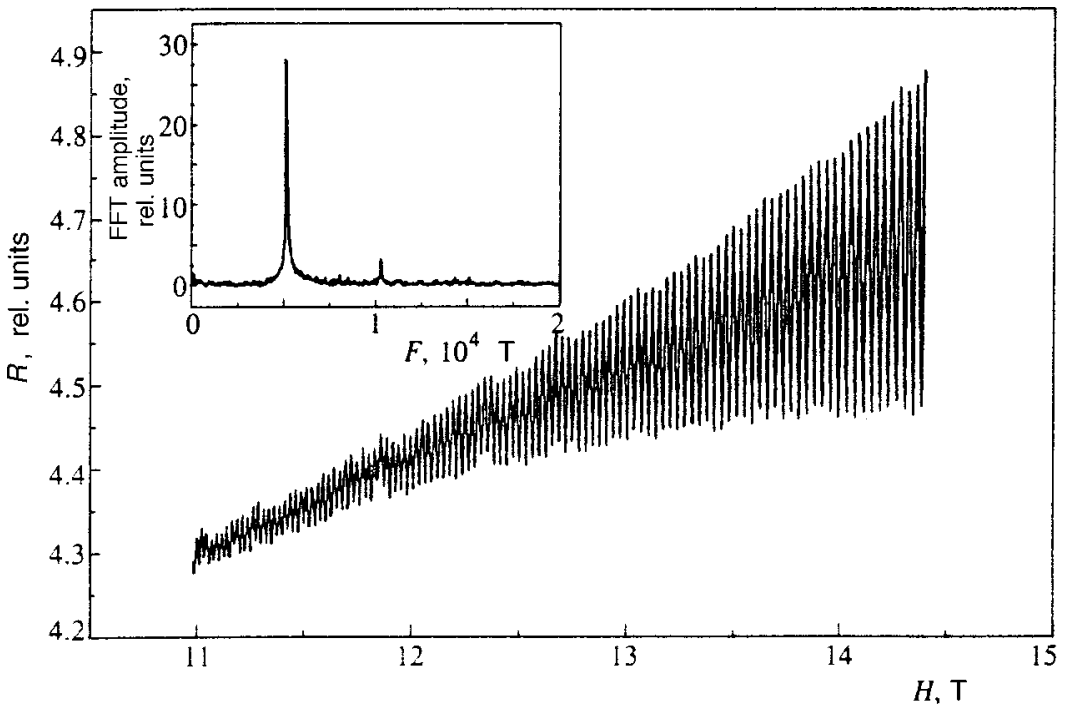

FIG. 1. Shubnikov-de Haas oscillations: $\theta=0^{\circ}, T$ $=1.45 \mathrm{~K}, \mathbf{I} \perp \mathbf{a b}$. The inset shows the FFT amplitude of these oscillations as a function of the frequency $F$ of the quantum oscillations. parameters of the quantum mechanical oscillations first observed in this compound ${ }^{3}$ confirms this level of filling of the band.

Data from an analysis of the composition and structure corresponding to the formula $(\mathrm{BO})_{2} \mathrm{Cl}\left(\mathrm{H}_{2} \mathrm{O}\right)_{3}$ were later presented, ${ }^{4}$ along with some results from a study of the reluctance of this complex that were consistent with the proposed formula.

Nevertheless, the chemical composition of BO chloride is still under discussion. A chemical formula of the form (BO) ${ }_{2} \mathrm{Cl}_{1.28}\left(\mathrm{H}_{3} \mathrm{O}\right)_{0.28}\left(\mathrm{H}_{2} \mathrm{O}\right)_{2.44}$ has been proposed, ${ }^{5}$ while fully retaining the structural motif proposed before. ${ }^{3,4}$ This difference in the description of the chemical composition does not, however, lead to a change in the degree of filling of the band, since the excess negative charge on the anion (associated with the chlorine) beyond that implied by the formula $(\mathrm{BO})_{2} \mathrm{Cl}\left(\mathrm{H}_{2} \mathrm{O}\right)_{3}$, is balanced by the positive charge on the $\mathrm{H}_{3} \mathrm{O}$.

In this paper we offer a more detailed investigation of the Shubnikov-de Haas and de Haas-van Alphen oscillations and semiclassical angular oscillations in the reluctance. These studies provide a more detailed idea of the size and shape of the Fermi surface in BO chloride, and make it possible to estimate the parameters of its electron system. The possible effect of the chemical composition of the anion on the behavior of these oscillations is not discussed here.

\section{EXPERIMENT}

Single crystal samples of $(\mathrm{BO})_{2} \mathrm{Cl}_{x}\left(\mathrm{H}_{2} \mathrm{O}\right)_{y}$ obtained in two chemical groups under differing synthesis conditions ${ }^{4,5}$ were used in the experiments. Nevertheless, the samples were shown to be identical by x-ray structural analysis. The samples consisted of irregular slabs with average dimensions of $1 \times 1 \times 0.1 \mathrm{~mm}^{3}$. The plane of the samples coincided with the crystallographic ab plane, which contains the conductive layers. These layers alternate along the short dimension of the samples, which coincides with the $\mathbf{c}^{*}$ axis.

The reluctance was measured by the standard four contact method using a $330 \mathrm{~Hz}$ alternating current. Here the measurement current $\mathbf{I}$ could be directed either in the ab plane or perpendicular to it. A magnetic field was created either by a superconducting solenoid with a maximum field of $15 \mathrm{~T}$ or by a resistive magnet with a field of up to $20 \mathrm{~T}$, and the minimum temperature was $0.45 \mathrm{~K}$. In the reluctance measurements the orientation of the sample was varied using a two-axis gimbal mount, which made it possible to rotate the sample in declination and in the azimuthal angle.

The de Haas-van Alphen effect was studied in terms of the dependence of the torque on the magnetic field. ${ }^{6}$ Here the maximum magnetic field was $14 \mathrm{~T}$ and the minimum temperature lay below $0.48 \mathrm{~K}$.

\section{RESULTS OF MEASUREMENTS}

The average conductivity of the test samples at room temperature in the conducting plane is $50 \Omega^{-1} \mathrm{~cm}^{-1}$. All the samples are characterized by a metallic variation in the reluctance as a function of temperature: as the temperature is reduced from room temperature to liquid nitrogen temperature, the reluctance decreases on average by a factor of a few dozen.

Figures 1 and 2 show the Shubnikov-de Haas and de Haas-van Alphen oscillations for a magnetic field directed almost perpendicular to the conducting plane. For this field direction there is a single fundamental frequency for the quantum oscillations $F_{0} \simeq 4900 \mathrm{~T}$ (see insets to Figs. 1 and 2 ). When the field deviates significantly from this direction, beats become appreciable in the fundamental frequency (see Figs. 3 and 4). Analysis of the quantum oscillations and fast Fourier transforms reveal the complex nature of these beats, which result from the mixing of three oscillations with similar frequencies (insets to Figs. 3 and 4), at least for some directions of the field.

The angular dependence of these frequencies for the de Haas-van Alphen oscillations is shown in Fig. 5. There, for each direction of the field, different symbols correspond to different frequencies. This curve fit well by the formula

$$
F(\theta)=\frac{4900}{\cos \theta}[\mathrm{T}],
$$




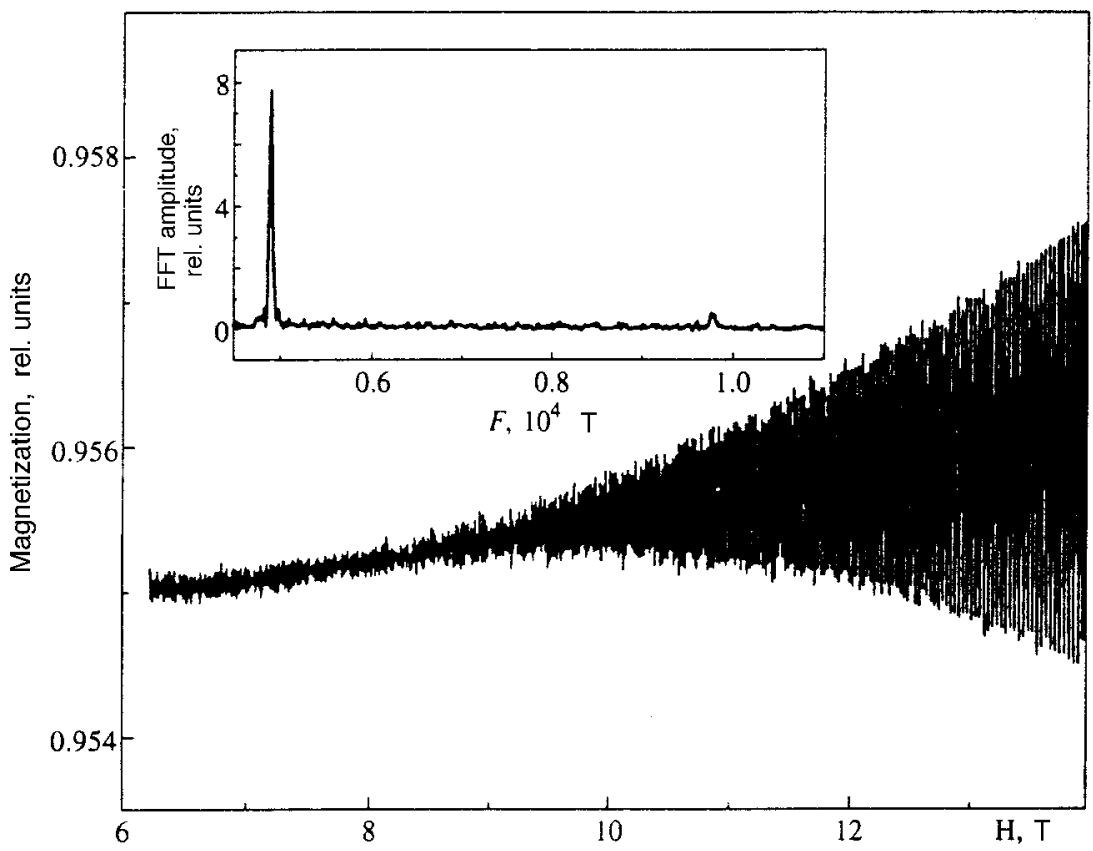

FIG. 2. De Haas-van Alphen oscillations: $\theta=4.7^{\circ}$, $T=0.5 \mathrm{~K}$. The inset shows the FFT of these oscillations. where $\theta$ is the angle between the field and the normal to the conducting plane. Here, recording several frequencies at each angle has essentially no effect on the curve, since the difference between the frequencies is never greater than a few percent and does not exceed the average scatter. Thus, we can assume that Eq. (1) yields the fundamental frequency to high accuracy.

Figure 6 illustrates the angular dependence of the amplitude of the de Haas-van Alphen oscillations. As in the case of the angular variation of the frequency, the amplitudes corresponding to oscillations at differing frequencies are indicated by different symbols. Despite the complicated angular dependence of the amplitude of the oscillations at different frequencies, the common zeroes in the amplitude at $\theta= \pm 41$ and $\pm 57^{\circ}$ are evident. The amplitude null at $\theta$ $=0^{\circ}$ is nonphysical and is related to the method for observing the de Haas-van Alphen oscillations. ${ }^{6}$ The amplitude nulls

at $\theta= \pm 41^{\circ}$ and $\theta= \pm 57^{\circ}$ are probably related to the fact that the decreasing spin factor in the Lifshitz-Kosevich formula ${ }^{7}$ vanishes in those directions, i.e.,

$$
R_{s}=\cos (p \pi g \mu / 2)=0,
$$

where $p$ is the harmonic number, $g$ is the $g$-factor, $\mu=m^{*} / m_{0}, m^{*}$ is the effective electron mass, and $m_{0}$ is the free electron mass.

The effective carrier mass was estimated on the basis of the temperature dependence of the amplitude of the quantum oscillations. Here the decreasing temperature factor was taken to have the form

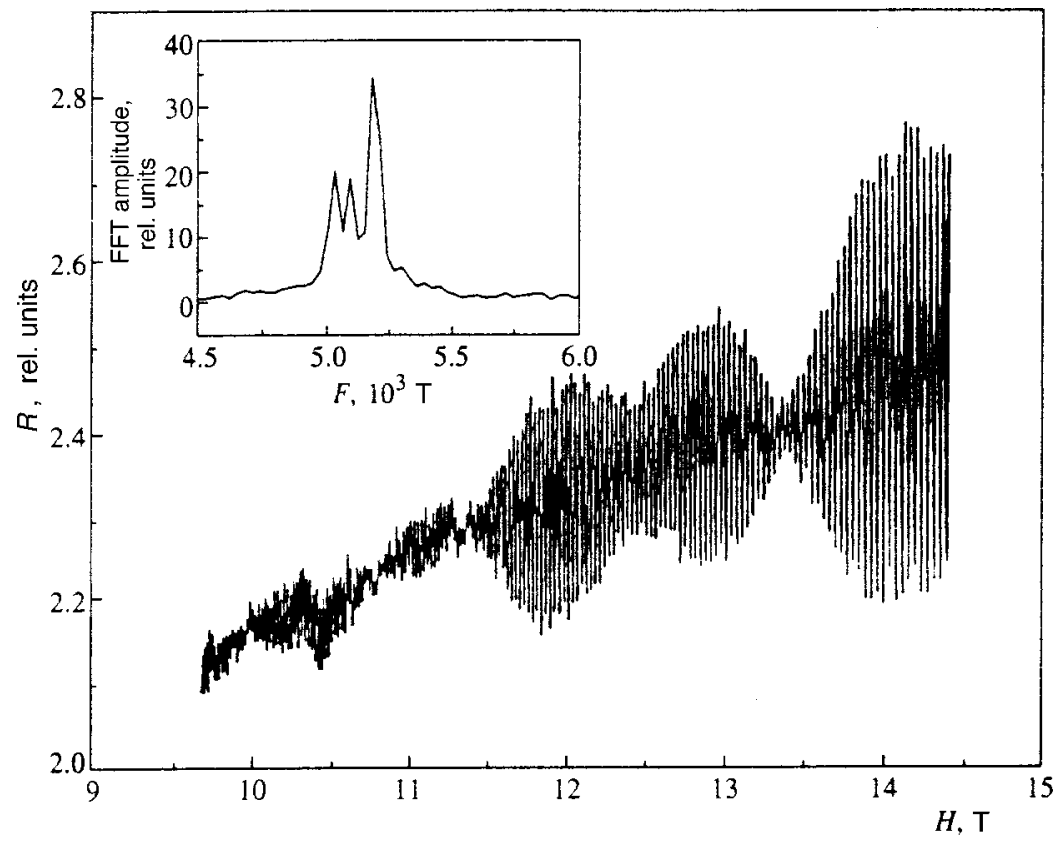

FIG. 3. Shubnikov-de Haas oscillations: $\theta=23^{\circ}$, $T=1.45 \mathrm{~K}, \mathbf{I} \perp \mathbf{a b}$. The inset shows the FFT of these oscillations. 


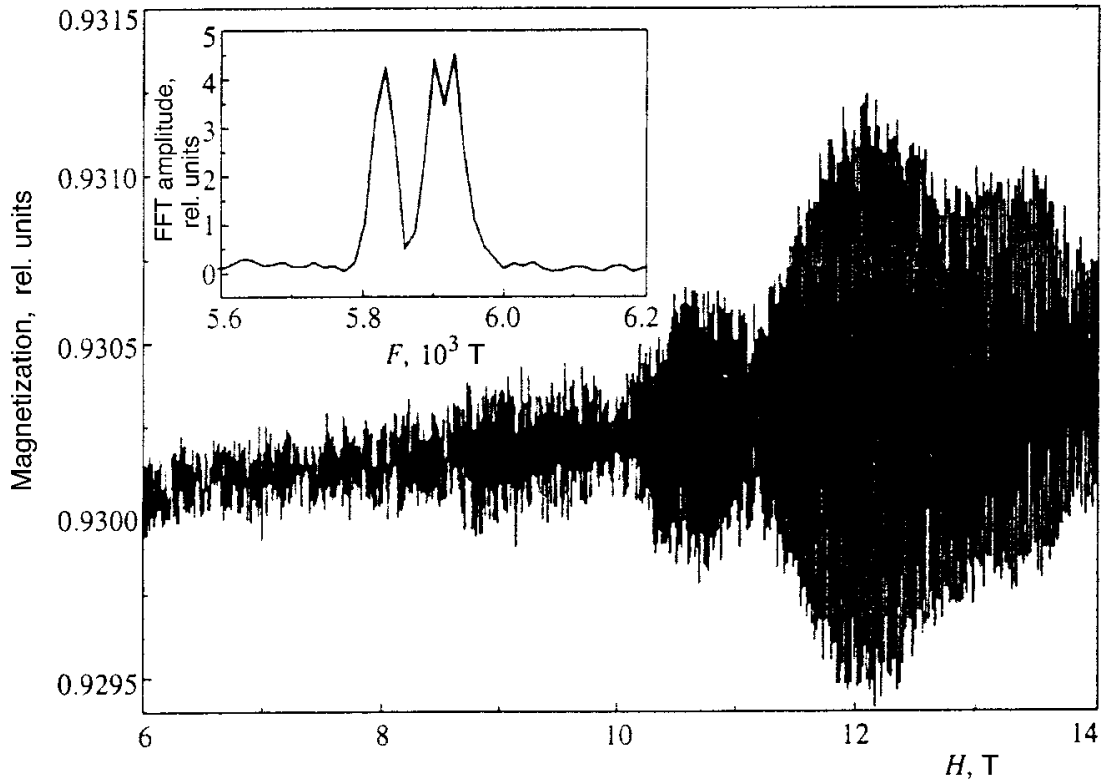

FIG. 4. De Haas-van Alphen oscillations: $\theta=34.7^{\circ}$, $T=0.5 \mathrm{~K}$. The inset shows the FFT of these oscillations.

$$
R_{t}=\frac{\alpha p \mu T / H}{\sinh (\alpha \mathbf{p} \mu T / H)},
$$

where $\alpha=2 \pi^{2} k_{B} m_{0} / e \hbar=14.7 \mathrm{~T} / \mathrm{K}, T$ is the temperature, and $H$ is the magnetic field. The mass in the conducting plane determined in this way for several of the samples had a substantial scatter and lay within the interval $m^{*}=(1.65-$ 2.0) $m_{0}$.

The Dingle temperature was determined from the fieldinduced variation in the amplitude of the quantum oscillations using a Dingle reducing factor of the form

$$
R_{D}=\exp \left(-\alpha p \mu T_{D} / H\right) \text {. }
$$

The Dingle temperature lies in the range $T_{D}=3-4 \mathrm{~K}$ for all samples measured.
Figure 7 shows the angular variation in the reluctance for a constant field of $14.3 \mathrm{~T}$. The maxima of the pronounced angular oscillations are periodic in $\tan \theta$. In Fig. 8, the period of these oscillations is plotted in polar coordinates as a function of the azimuthal angle $\varphi$ in the conducting plane. Clearly the period is essentially independent of the azimuthal angle, and is $\Delta \approx 0.5$.

\section{DISCUSSION}

The crystal lattice parameters of BO chloride were first given in Ref. 2 and confirmed in Refs. 4 and 5. The band structure of this material was calculated on the basis of these data and found to be in agreement with preliminary observations of quantum oscillations. ${ }^{3}$ According to this calculation,

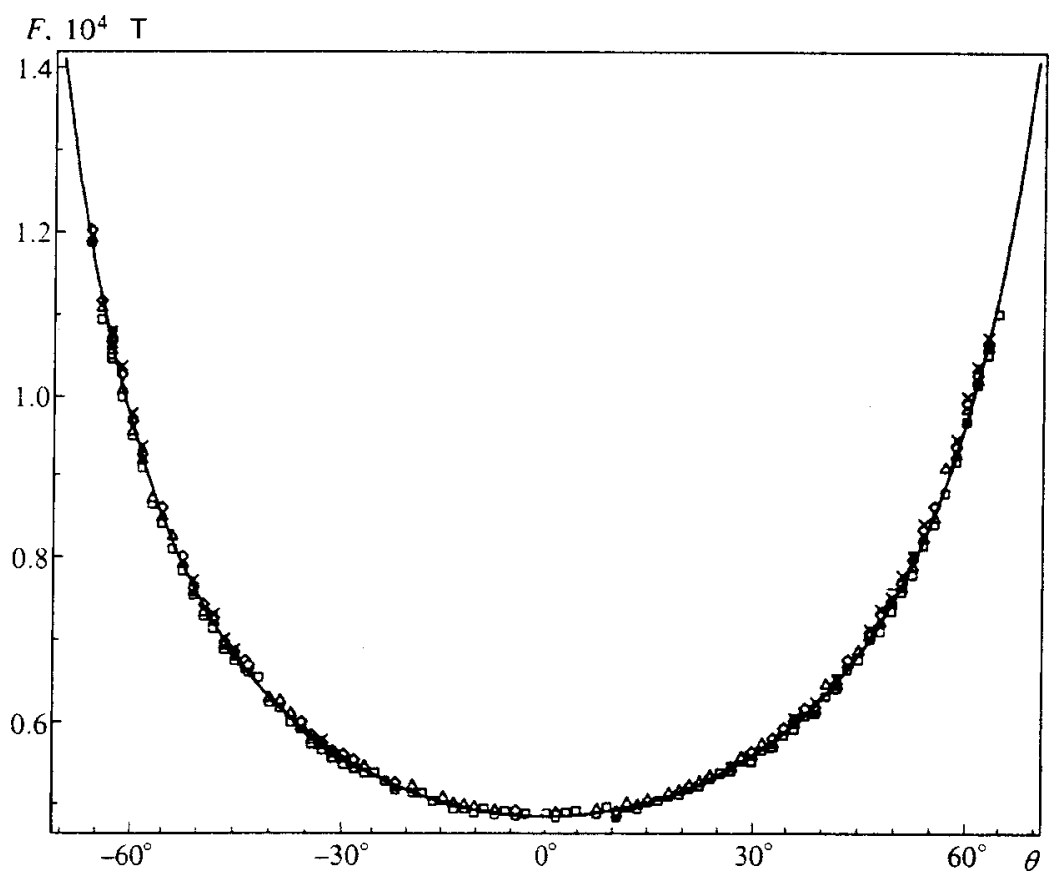

FIG. 5. Angular dependence of the frequency of de Haas-van Alphen oscillations; $T=0.5 \mathrm{~K}$. The smooth curve corresponds to $F(\theta)=4900 / \cos \theta[\mathrm{T}]$. 


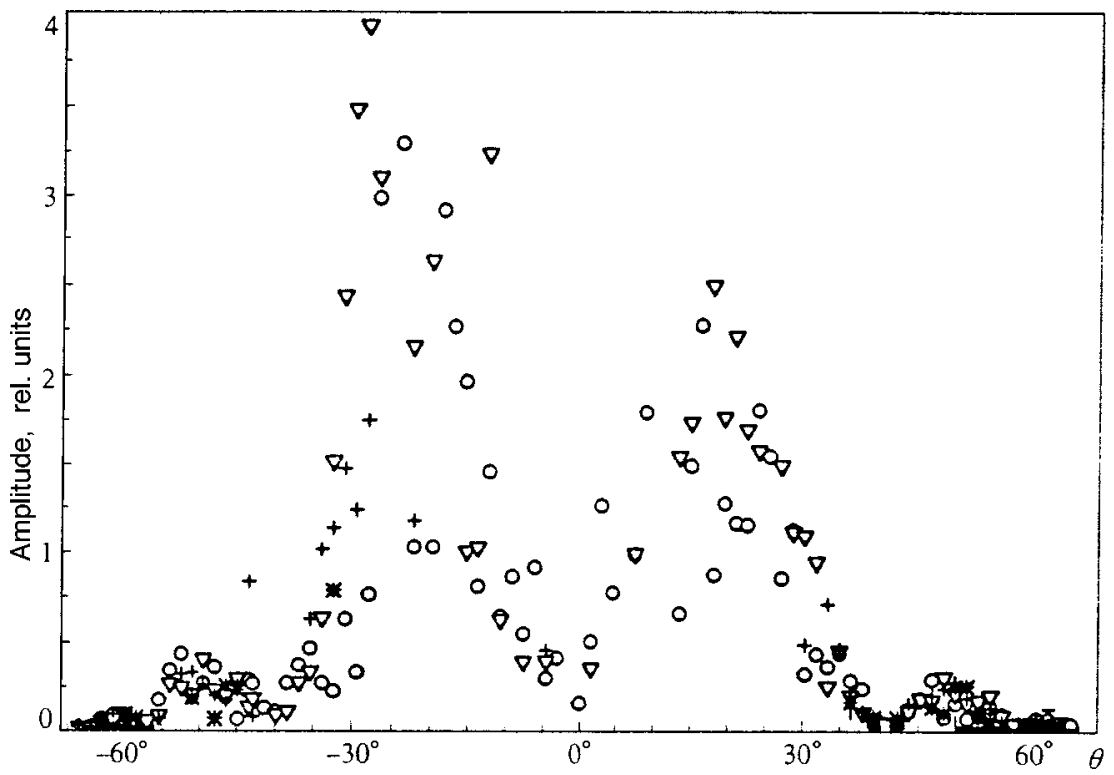

FIG. 6. Angular dependence of the amplitude of the de Haas-van Alphen oscillations; $T=0.5 \mathrm{~K}$. the Fermi surface (Fig. 9) in $(\mathrm{BO})_{2} \mathrm{Cl}_{x}\left(\mathrm{H}_{2} \mathrm{O}\right)_{y}$ is a cylinder with its axis oriented in the $\mathrm{c}^{*}$ direction. The cross section of this cylinder in the ab plane coincident with the plane of best conductivity is a perfect circle whose area is roughly half that of the corresponding cross section of the Brillouin zone.

For all the $\mathrm{BO}$ chloride crystals studied here, the frequency of the quantum oscillations is $F_{0} \approx 4900 \mathrm{~T}$ for a field $\mathbf{H} \perp \mathbf{a b}$, which corresponds to $50 \%$ of the area transected in the first Brillouin zone, and agrees well with theoretical calculations. The angular dependence of the frequency of these oscillations is fit well by Eq. (1) and corresponds to a cylindrical Fermi surface oriented along $\mathbf{c}^{*}$.

The angular oscillations in the reluctance, with peaks periodic in $\tan \theta$ (Fig. 7), are also related to the motion of carriers over the cylindrical Fermi surface, and the cylinder must be weakly corrugated along its axis. ${ }^{8,9}$ The period of oscillations in the reluctance is independent of azimuthal angle in BO chloride crystals (Fig. 8), so the cross section of the cylinder can be represented as a perfect circle in the ab plane. In this case, the Fermi momentum $k_{F}$ is related to the period $\Delta$ of angular oscillations in the reluctance by the simple formula ${ }^{8}$

$$
\Delta=\pi / k_{F} d,
$$

where $d$ is the distance between conducting planes. The estimated cross section $S=\pi k_{F}^{2} \simeq 3 \times 10^{15} \mathrm{~cm}^{-2}$ is in good agreement with theoretical calculations and the quantum oscillation data. Thus, the quantum and semiclassical oscillations in the organic metal $(\mathrm{BO})_{2} \mathrm{Cl}_{x}\left(\mathrm{H}_{2} \mathrm{O}\right)_{y}$ correspond to a weakly corrugated cylindrical Fermi surface with a perfectly circular cross section of radius $\simeq 3 \times 10^{7} \mathrm{~cm}^{-1}$ in the $\mathbf{a b}$ plane.

The slight corrugation in the cylindrical sheet of the Fermi surface must result in the existence of several similar
$R$, rel. units

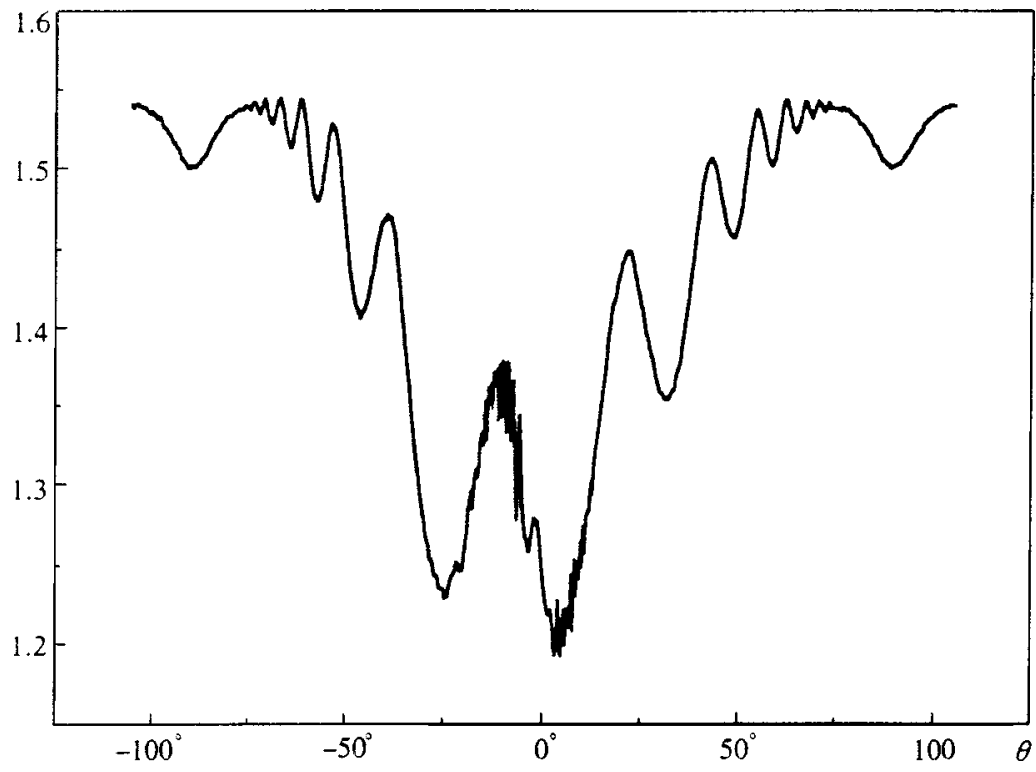

FIG. 7. Angular dependence of the reluctance; $T=0.5 \mathrm{~K}$, $H=14.3 \mathrm{~T}, \mathbf{I} \perp \mathbf{a b}$. 


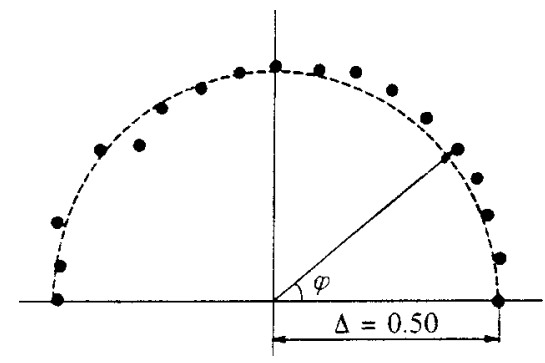

FIG. 8. The period of angular oscillations in the reluctance as a function of the azimuthal angle $\varphi$ in polar coordinates.

extremal cross sections (in the case of simple corrugation, there should be two), and this may show up as beats in the fundamental frequency. In the compound studied here, beats are observed for field directions not coincident with $\mathbf{H} \| \mathbf{c}^{*}$ (Figs. 3 and 4). These beats, however, have a complex shape, since they result from combining more than two oscillations at differing frequencies. We might assume a complicated corrugation with several differing extremal cross sections, but one would then scarcely expect the pronounced angular oscillations in the reluctance observed in the sample crystals. In addition, there is still no explanation for the lack of any beats whatsoever for a field with $\mathbf{H} \| \mathbf{c}^{*}$.

The beats might be related to crystal imperfections of the samples, such as twinning, intergrowth, etc. This explanation, however, can be ruled out by direct $\mathrm{x}$-ray analysis of the test samples, which confirms that they are high-quality single crystals.

The distorted form of the quantum oscillations (including beats) might arise through magnetic interaction, ${ }^{7}$ but the latter is only significant when the absolute amplitude of oscillations in the magnetic moment $\mu_{B} \mathbf{M}$ is comparable to the period $H^{2} / F$ of the oscillations. In the test samples,

$$
\mu_{B} M \approx 2.5 \mathrm{G} \ll 200 \mathrm{G} \approx H^{2} / F,
$$

where $\mu_{B} M \approx 2.5 \mathrm{G}$ is the maximum attainable absolute amplitude in the experiments with a $10 \mathrm{~T}$ field, so magnetic interaction can be neglected.

Finally, there is yet another possible reason for the beats. The calculated Fermi surface in Ref. 5 differs somewhat from that in Fig. 9. The cylindrical sheet intersects the boundary of the Brillouin zone near the point $X$, rather than being tangent to it, forming very small pockets at this site. This can lead to the coexistence of a small orbit with the main orbit, which corresponds to the fundamental frequency. We can then expect sum and difference frequencies to appear, including some near the fundamental. This latter scenario would seem to be the most plausible, but it is obviously very vulnerable at present and requires more convincing confirmation and additional study.

The condition (2) for the appearance of "spin nulls" at $\theta \approx \pm 41$ and $\pm 57^{\circ}$ is satisfied by the first harmonic, assuming that the effective mass and angle of inclination are related by the characteristic formula for a cylindrical Fermi surface, $\mu(\theta)=\mu(0) / \cos \theta$. Here the splitting factor is $S_{s}$ $=g \mu(0) / 2 \approx 1.85$. Given the spread in the estimate of the effective mass obtained from the temperature dependences of the amplitude of the quantum oscillations, $m=(1.65-$ 2.0) $m_{0}$, the $g$ factor becomes $g=1.85-2.23$. The $g$-factor determined in this way usually includes corrections owing to many-particle interactions, which makes it different from the free electron $g$-factor, $g_{0}=2 .^{7}$ The closeness of $g$ and $g_{0}$ argues for the weakness of these interactions. In the test samples, any assumptions regarding the influence of manyparticle interactions would be incorrect because of the excessive error in determining the $g$-factor due to the spread in the value of the effective mass. More detailed experiments will be required to make the latter more accurate.

\section{CONCLUSION}

We have studied the behavior of the magnetic moment and reluctance of a group of samples of the organic metal (BO) ${ }_{2} \mathrm{Cl}_{x}\left(\mathrm{H}_{2} \mathrm{O}\right)_{y}$. Shubnikov-de Haas and de Haas-van Alphen quantum oscillations and semiclassical angular oscillations in the reluctance were observed. An analysis of these data indicate that the Fermi surface of this compound is a cylinder weakly corrugated along its axis, and is a perfect circle of radius $k_{F} \simeq 3 \times 10^{7} \mathrm{~cm}^{-1}$ perpendicular to the axis. These results agree with theoretical calculations. Complicated beats involving the fundamental frequency of the quantum oscillations were observed, but their nature is as yet unclear.

We thank L. P. Rozenberg for the X-ray structural data, and E. B. Yagubskiǔ and R. P. Shibaeva for useful discussions. This work was supported by the Russian Fund for

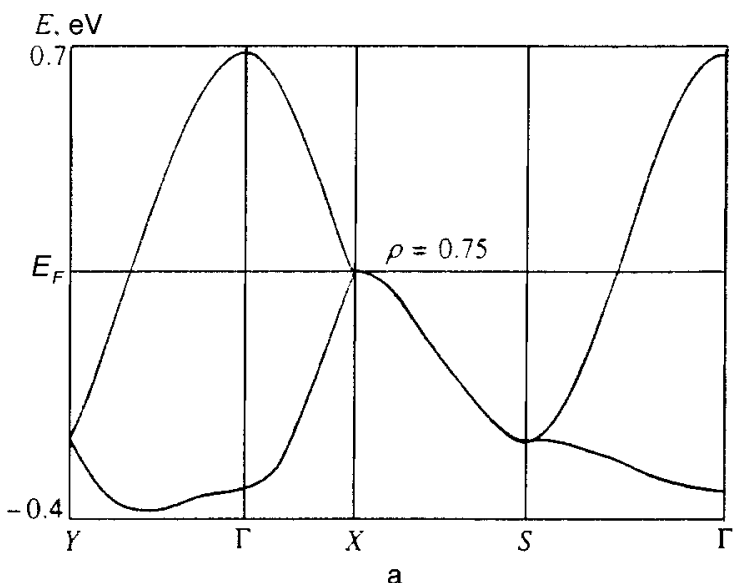

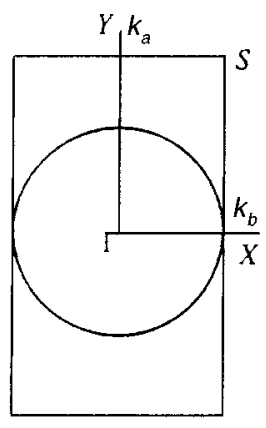

FIG. 9. The energy band structure (a) and Fermi surface (b) in the organic metal $(\mathrm{BO})_{2} \mathrm{Cl}\left(\mathrm{H}_{2} \mathrm{O}\right)_{3}{ }^{3} \cdot$ 
Fundamental Research (Grants No. 96-02-18957 and No. 9602-17475), the State Scientific Program on Statistical Physics, Grant 1/70 206 from the Volkswagen-Stiftung, a Grant from NWO, and Grant No. INTAS-93 2400 EXT.

*)E-mail: pesot@icp.ac.ru

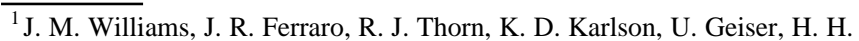
Wang, A. A. Kini, and M. H. Whangbo, Organic Superconductors: Synthesis, Structure, and Theory, Prentice-Hall, Englewood Cliffs, NJ (1992).

${ }^{2}$ D. Schweitzer, S. Kahlich, I. Heiman, S. E. Lan, B. Nuber, H. J. Keller, K. Winzer, and H. W. Helberg, Synth. Met. 56, 2827 (1993).

${ }^{3}$ T. Mori, S. Oshima, H. Okuno, K. Kato, H. Mon, and S. Tanaka, Phys. Rev. B 51, 11110 (1995).
${ }^{4}$ E. I. Zhilyaeva, S. A. Torunova, R. N. Lyubovskaya, S. V. Konovalikhin, O. A. Dyachenko, R. B. Lyubovskii and S. I. Pesotskii, Synth. Met. 83, 7 (1996).

${ }^{5}$ R. P. Shibaeva, S. S. Khasanov, B. Zh. Narymbetov, L. V. Zorina, L. P. Rozenberg, A. V. Bazhenov, N. D. Kusch, E. B. Yagubskii, C. Rovira, and E. Canadell, submitted to J. Mat. Chem.

${ }^{6}$ P. Christ, W. Biberacher, H. Muller, and K. Andres, Solid State Commun. 91, 451 (1994).

${ }^{7}$ D. Shoenberg, Magnetic Oscillations in Metals, Cambridge Univ. Press, Cambridge (1984).

${ }^{8}$ Y. K. Yamaji, J. Phys. Soc. Jpn. 58, 1520 (1989).

${ }^{9}$ M. V. Kartsovnik, V. N. Laukhin, S. I. Pesotskii, I. F. Schegolev, and V. M. Yakovenko, J. de Phys. I 2, 89 (1992).

Translated by D. H. McNeill 